\title{
Low cost ionic liquid-water mixtures for effective extraction of carbohydrate and lipid from algae
}

\author{
Trang Q. To, ${ }^{\text {aa }}$ Kerryn Procter, ${ }^{\text {a }}$ Blake A. Simmons, (D) ${ }^{\mathrm{b}}$ \\ Suresh Subashchandrabose ${ }^{c}$ and Rob Atkin (D) ad
}

Received 1st May 2017, Accepted 17th May 2017

DOI: $10.1039 / c 7 f d 00158 d$

\begin{abstract}
Biomass based biofuels are already an important energy source, and will increasingly be so in the future as the need for renewable energy rises. Due to their fast multiplication rates, algae can provide a sustainable supply of biomass, and are attractive because they do not compete with food crops for habitat. Here we show that biomass derived from Chlorella vulgaris and Spirulina platensis can be pretreated with low cost choline amino acid based ionic liquids to effectively yield lipids (30.6\% and $51 \%$ total lipids) and sugars (71\% and $26 \%$ total sugars). The ionic liquids dissolve the lipids, leaving behind a carbohydrate rich solid. The lipids were extracted with hexane, and the solid was subjected to enzyme hydrolysis to release fermentable sugars. These results open new pathways towards the dual production of biodiesel and bioethanol from algae, using low cost ionic liquids.
\end{abstract}

\section{Introduction}

As world fossil fuel resources begin to dwindle, alternative clean fuel production from renewable sources is not only preferred, but will also become compulsory in the future. In recent years, there has been increasing interest towards using biomass as a renewable energy source. Among the many types of biomass available, microalgae have gained attention for biodiesel production, due to their high lipid content. ${ }^{1-5}$ Microalgae are ubiquitous photosynthetic organisms that live in water and do not compete with food crops in terms of land and nutrient use. They harvest energy from the sun, $\mathrm{CO}_{2}$, and nutrients and minerals in the water environments in which they reside to synthesise a variety of complex macromolecules including carbohydrates, lipids and proteins. Microalgae can grow on wastewater that is rich in proteins and inorganic nitrogens, and so are used

${ }^{a}$ Priority Research Centre for Advanced Fluids and Interfaces, Newcastle Institute for Energy and Resources, Australia. E-mail: Trang.To@newcastle.edu.au

${ }^{b}$ Lawrence Berkeley National Laboratory, Joint BioEnergy Institute, Berkeley, CA, 94720, USA

${ }^{c}$ Global Centre for Environmental Remediation, Faculty of Science, University of Newcastle, Callaghan, New South Wales 2308, Australia

${ }^{d}$ School of Molecular Sciences, The University of Western Australia, WA 6009, Australia 
extensively in wastewater remediation; ${ }^{6,7}$ a short culture period of 14 days is sufficient for algae to remove up to $70-90 \%$ of nitrogen, phosphorus and COD (chemical oxygen demand) from winery and dairy wastewater. ${ }^{6}$ Microalgae are ideal platforms for biodiesel production due to their rapid multiplication rates and ability to accumulate a large mass of lipids that are readily converted through transesterification. Under suitable culture conditions, microalgae can accumulate up to $70 \%$ of lipids in dry biomass. ${ }^{8,9}$ Other than lipids, microalgae also contains significant amounts of carbohydrates (cellulose and starch), which can be converted to ethanol for further biofuel production. However, most studies on algae have focused solely on the lipids, with the carbohydrates neglected entirely.

The most popular method to extract lipids from microalgae is Soxhlet extraction using hexane, ${ }^{10}$ but this approach has several disadvantages in terms of commercial viability. First, the cell walls of microalgae are made up of a highly complex matrix of polysaccharides intercalated with proteins, ${ }^{11-13}$ which has a high chemical resistance to non-polar solvents. Second, hexane is incapable of extracting lipids stored in lipid droplets, as it cannot cross the (protein bound) polar phospholipid-membrane. On the other hand, polar solvents such as methanol/chloroform cross the phospholipid barrier ${ }^{2}$ by diffusion and extract these lipids (the Bligh \& Dyer method ${ }^{\mathbf{1 4}}$ ). Physical pretreatment to break open the cells (such as osmotic shock, ${ }^{15}$ blending, microwave or laser ${ }^{16}$ ) followed by the extraction of lipids with solvents has been investigated, but these physical pretreatment steps, together with the need for dry algal biomass in the extraction, result in energy intensive processes that are not commercially feasible..$^{2,3,17}$

Chemical pretreatment offers a potential alternative that may be commercially viable. For example, a biphasic system of acidic solution and hexane in a bioreactor $\left(155^{\circ} \mathrm{C}\right)$ facilitates the recovery of up to $97 \%$ of lipids and the release of $90 \%$ glucose from Chlorella and Scenedesmus. ${ }^{18}$ Certain ionic liquids (ILs), which consist entirely of cations and anions, ${ }^{19-21}$ are interesting candidates for the pretreatment step due to their favourable physical properties. ILs are tuneable, have the capacity to dissolve a wide range of materials, and low toxicity variants are known. ${ }^{20,21}$ ILs have attracted research interest for the pretreatment of lignocellulosic biomass for enhanced bioethanol production. ${ }^{22,23}$ Certain ILs selectively dissolve the lignin component of the lignocellulosic biomass, leaving behind a cellulose-rich residue easily digested by enzymes to release fermentable sugars.

The use of ILs for the pretreatment of algal biomass for lipid extraction has been investigated previously. ${ }^{24-29}$ ILs work by dissolving the lipids, which separate out of the liquor on addition of an antisolvent such as methanol, which are then recovered using hexane. However, improvement is needed in several areas. ${ }^{24}$ Most of the ILs employed in these studies are imidazolium- and pyridinium-based, which are 'classic' ILs but are expensive and toxic. Only two studies so far have used the cheaper ammonium- and phosphonium-based ILs. ${ }^{29,30}$ However, regardless of the cation type employed the algal biomass solid loading was low (between 5 and $10 \mathrm{wt} \%$ ), ${ }^{31}$ and only a few studies have attempted to recover other valuable components of the algal biomass, such as carbohydrates and carotenoids. $^{32-34}$

This study explores the use of a series of cheap and environmentally benign ILs for the pretreatment of microalgae. Made from simple acid-base reactions between two naturally occurring non-toxic chemicals, choline (an ammonium) 
and amino acids, these ILs are significantly cheaper and less toxic than imidazolium-based ILs. To further reduce the cost, instead of using neat ILs, mixtures of IL-water are utilised. This class of ILs has been shown to perform well for lignocellulosic biomass processing, ${ }^{35}$ and facilitate a one-pot reaction from biomass to ethanol. Two distinct, representative, microalgae are probed, one from the prokaryotic cyanobacterial group, Spirulina platensis, and the other from the eukaryotic green algae Chlorella vulgaris. The latter species $C$. vulgaris is extensively studied due to its high lipid content ( $300 \mathrm{mg} \mathrm{g}^{-1}$ dry cell). However, $C$. vulgaris possesses a robust and complex cell wall, which makes it resistant to chemical attack. In order to examine whether our ILs can penetrate this barrier, two samples of $C$. vulgaris were investigated, one with an intact cell wall, and one with a cracked cell wall. High solid loading ( $20 \mathrm{w} / \mathrm{v} \%$ ), moderate temperature $\left(70{ }^{\circ} \mathrm{C}\right)$ and a short reaction time $(3 \mathrm{~h})$ were employed and both lipids and carbohydrates were recovered.

\section{Materials and methods}

\section{Synthesis of ionic liquids}

1-Ethyl-3-methylimidazolium acetate, choline hydroxide (46 wt\% in water), all amino acids, namely L-arginine, glycine, L-lysine, and L-phenylalanine were purchased from Sigma Aldrich and used without any further purification. In the synthesis equal amounts (by mol\%) of choline hydroxide and amino acids were mixed together. The resultant mixtures were heated up to $70{ }^{\circ} \mathrm{C}$ and stirred for 3 hours. Water was added to the mixture during heating to ensure that the amino acids had completely dissolved. Table 1 shows the structure of the ionic liquids, along with their water content; note that as natural biomass contains large volumes of water, the ILs were not dried prior to use.

\section{Pretreatment of algae with ILs}

Biomass from two strains, Chlorella vulgaris (Algomed Germany, certified additive-free and untreated) and Spirulina platensis (Nourishme Organics) were sourced commercially. Food grade Chlorella vulgaris (cell wall broken by heating) was also obtained (Synergy Natural).

$0.5 \mathrm{~g}$ of the biomass samples was pretreated by dissolving in $2.5 \mathrm{~mL}$ of the selected ILs at $70{ }^{\circ} \mathrm{C}$ for 3 hours with continuous stirring. Ten $\mathrm{mL}$ of water was added and the samples were centrifuged to separate the solid and liquid fractions (6000 rpm, 15 minutes). The pretreated biomass solid was subjected to further washing with water $(3 \times 10 \mathrm{~mL}$ water $)$ to remove residual IL.

\section{Extraction of lipids from the IL liquors}

The liquid fractions from centrifugation were acidified with concentrated hydrochloric acid (37 wt\%). Acidification converts fatty acids to their neutral form that is more soluble in hexane. The acid was added dropwise until the solution reached $\mathrm{pH} 3$. The lipids were extracted three times with hexane $(3 \times 5 \mathrm{~mL})$, each time the centrifuge tubes were vigorously vortexed (15 seconds) followed by centrifugation (6000 rpm, 30 minutes). The hexane layers were collected and combined. Finally, hexane was removed under vacuum to yield crude lipid that 


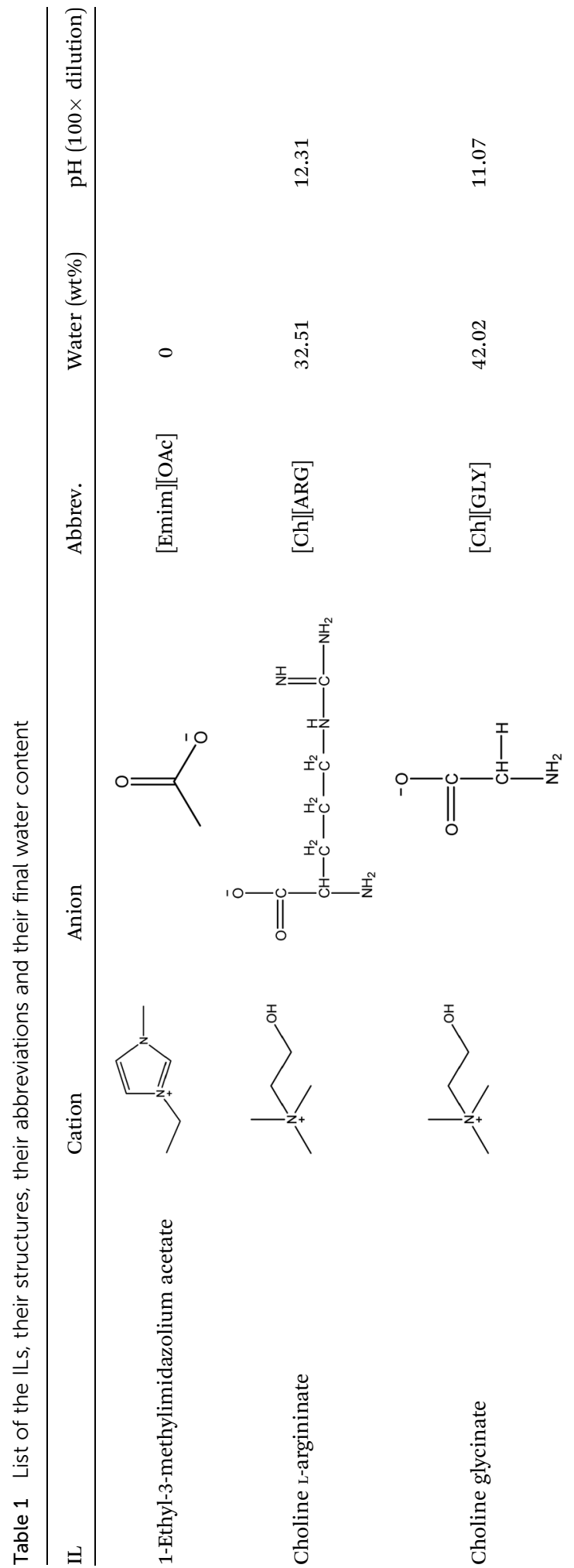

Paper

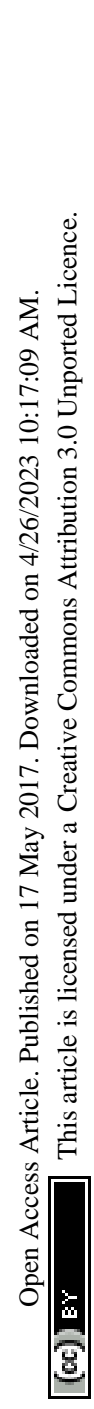

96 | Faraday Discuss., 2018, 206, 93-112 This journal is (C) The Royal Society of Chemistry 2018 


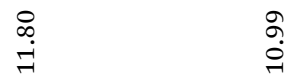

$\begin{array}{ll}\stackrel{0}{1} & \stackrel{7}{\infty}\end{array}$

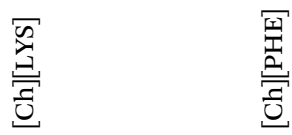

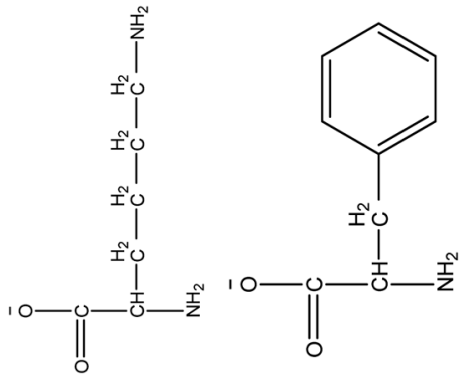<smiles>[CH]CC[C+](C)(C)C</smiles><smiles>[CH2]CC[C+](C)(C)C</smiles>

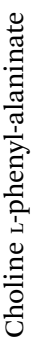


was weighed using an analytical balance. The composition of the crude was analyzed to determine its purity and to calculate the actual lipid yield.

\section{Compositional analysis of the algal biomass and extracted crude lipids from the IL liquor}

The original biomass and their solid fractions from centrifugation were analyzed to determine the total lipids, carbohydrate content, total solids and ash content. The crude lipids extracted from the IL liquors were analyzed for purity.

Lipids. Total lipid content was determined as Fatty Acid Methyl Esters (FAME) using in situ transesterification according to the laboratory protocol developed by the National Renewable Energy Laboratory (NREL). ${ }^{36}$ In a GC glass vial, algal biomass or crude lipid ( $\sim 10 \mathrm{mg})$ was added together with chloroform/methanol $(200 \mu \mathrm{L} 2: 1 \mathrm{v} / \mathrm{v}), \mathrm{HCl}(0.6 \mathrm{M}$ in methanol, $600 \mu \mathrm{L})$ and $2.645 \mathrm{mg}$ of tridecanoic acid methyl ester that serves as the internal standard (C13:0ME, Sigma Aldrich, 25 $\mu \mathrm{L}$ of $10.58 \mathrm{mg} \mathrm{mL}^{-1}$ stock solution in hexane). The mixture was vortexed vigorously for 5 minutes before incubation at $85{ }^{\circ} \mathrm{C}$ for one hour, with brief vortexing every 15 minutes. The mixture was then left to cool to room temperature and $1 \mathrm{~mL}$ of hexane was added. The vial was vortexed again and left to stand for one hour. The hexane layer was extracted and sent for gas chromatography-mass spec (GCMS) analysis.

The GC program (Shimadzu machine fitted with a Restek Rxi-5Sil MS column) was as follows: $1 \mu \mathrm{L}$ injection at $8: 1$ split ratio, inlet temperature of $250{ }^{\circ} \mathrm{C}$; constant flow of $1 \mathrm{~mL} \mathrm{~min}{ }^{-1}$ helium; oven temperature: $100{ }^{\circ} \mathrm{C}$ for $1 \mathrm{~min}, 25^{\circ} \mathrm{C}$ $\min ^{-1}$ up to $200{ }^{\circ} \mathrm{C}$ and hold for $1 \mathrm{~min}, 5^{\circ} \mathrm{C} \mathrm{min}^{-1}$ up to $250{ }^{\circ} \mathrm{C}$ and hold for $7 \mathrm{~min}$ (23 min total). For the first run, a full scan MS was carried out to identify the structure and the retention time of the FAMEs present. Selected ion monitoring (SIM) was then performed for enhanced sensitivity and accurate quantification. Calibration curves were created from standards made from a C8-C24 FAME mix (Supelco) and the C13:0ME internal standard. The Supelco mix covers most of the FAMEs present in the algal biomass, except for C16:2 and C16:3; whose quantification had to be done using the responsive factors obtained for C18:2n6 and C18:3n3 (present in the Supelco mix) as recommended in the NREL protocol.

Sugars. The total carbohydrate content was determined using a two-step sulphuric acid hydrolysis to hydrolyse the polymeric forms of the carbohydrates in the algal biomass into monomeric subunits according to the laboratory protocol developed by NREL. ${ }^{37}$ Algal biomass ( $25 \mathrm{mg}$ ) was added to $250 \mu \mathrm{L}$ of $72 \%$ (w/w) sulphuric acid, placed in a water bath at $30{ }^{\circ} \mathrm{C}$ for $1 \mathrm{~h}$ and stirred every 15 minutes with a glass rod to prevent aggregation. Water $(7 \mathrm{~mL})$ was added to the reaction tube and the sample was incubated in an oven at $121^{\circ} \mathrm{C}$ for $1 \mathrm{~h}$. The resulting solution was neutralized with $\mathrm{CaCO}_{3}$ (to $\mathrm{pH}$ 6) and centrifuged (13 $000 \mathrm{rpm}, 30$ seconds). The supernatant was collected and its carbohydrate concentration was measured using a ACCU-CHEK Performa Blood Glucose Meter (Roche Diagnostics).

Moisture, total solid and ash. Moisture, total solid and ash in the algal biomass were determined from the thermal behaviour of the algal biomass using Thermal Gravimetric Analysis (TGA). Each sample (5-10 mg) was placed in an alumina crucible and heated from $30^{\circ} \mathrm{C}$ to $600^{\circ} \mathrm{C}$ with a ramp rate of $10^{\circ} \mathrm{C} \mathrm{min}^{-1}$, under a flow of $100 \mathrm{~mL} \mathrm{~min}^{-1}$ air. Moisture was determined as the first segment of 
weight loss (stabilized around $120-200{ }^{\circ} \mathrm{C}$ ), ash was determined as the weight remaining at the end, and total solids were calculated by subtracting the moisture and ash.

\section{Saccharification by enzyme hydrolysis}

An enzyme hydrolysis procedure was designed to target the cellulose and starch present in microalgae. The procedure was adopted from two standard protocols, the NREL protocol for lignocellulosic biomass ${ }^{38}$ and the Sigma Starch Assay protocol (STA-20). ${ }^{39}$

In a GC vial, algal biomass ( $20 \mathrm{mg})$ was mixed together with $40 \mu \mathrm{g}$ of tetracycline ( $4 \mu \mathrm{L}$ of $10 \mathrm{mg} \mathrm{mL}^{-1}$ solution in EtOH), $30 \mu \mathrm{g}$ of cycloheximide ( $3 \mu \mathrm{L}$ of $10 \mathrm{mg} \mathrm{mL} \mathrm{m}^{-1}$ solution in water), $0.6 \mu \mathrm{L}$ of cellulase (from Aspergillus sp., Sigma, equivalent to $25 \mathrm{U}$ per $\mathrm{g}$ of biomass), $2 \mu \mathrm{L}$ of $\alpha$-amyloglucosidase (from Bacillus licheniformis, Sigma, equivalent to $25 \mathrm{U}$ per $\mathrm{g}$ of biomass), $2 \mu \mathrm{L}$ of $\alpha$-amylase (from Aspergillus niger, Sigma, equivalent to $25 \mathrm{mg}$ protein per $\mathrm{g}$ of biomass) and $970 \mu \mathrm{L}$ of buffer solution ( $\mathrm{NaOAc} / \mathrm{AcOH}, \mathrm{pH}$ ). The vial was vortexed briefly then left at $50{ }^{\circ} \mathrm{C}$ with stirring for $24 \mathrm{~h}$. The D-glucose and D-galactose content were measured using the Accu-check Performa Glucose meter.

\section{Results and discussion}

IL pretreatment of biomass was carried out with a high loading of $20 \mathrm{w} / \mathrm{v} \%$ biomass/IL under very mild conditions $\left(70{ }^{\circ} \mathrm{C}\right.$ for $\left.3 \mathrm{~h}\right)$. Five ionic liquids were tested in this study: four choline amino acid ILs together with a conventional IL, 1-ethyl-3-methylimidazolium acetate ([Emim][OAc]) (structures in Table 1). [Emim][OAc] is a benchmark IL for lignocellulosic biomass pretreatment. ${ }^{22,40}$ When [Emim $][\mathrm{OAc}]$ is used neat, cellulose is preferentially dissolved in the IL and then recovered in solid form using an antisolvent such as water or methanol. The recovered solid contains mostly cellulose with less lignin and hemicellulose than the starting material. This process is defined as the dissolution process. ${ }^{22}$ When [Emim][OAc] is used in a mixture with water (the Ionosolv process), lignin is preferentially dissolved, leaving behind a cellulose rich solid. ${ }^{40}$ The Ionosolv process reduces the amount of IL required. The choline amino ILs in this study were used as aqueous solutions (around 65\% IL to $35 \% \mathrm{H}_{2} \mathrm{O}$, Table 1), i.e. following the Ionosolv concept, and are significantly cheaper in terms of starting materials and synthesis labour than [Emim][OAc]; synthesis is achieved by simply mixing choline hydroxide solution with an equivalent amount of amino acid to yield the IL within 3 hours without side products. The resultant IL water solutions were basic ( $\mathrm{pH}$ 11-12.3).

\section{Moisture, solid, ash and TGA profiles}

The main components of algae biomass are moisture, carbohydrates, proteins, lipids and ash. The TGA profiles of the three algae samples display similar composition, with $6-8 \%$ water, $85-89 \%$ solid and $3-7 \%$ ash (Table 2 , Fig. $1-3$ ). In addition to the primary mass loss profiles, the first derivative curves (dweight/ dtemperature) were also analysed to better identify the mass loss sections. The first derivative curve of untreated $C$. vulgaris and $S$. platensis displayed three main peaks, corresponding to three stages of decomposition: dehydration $\left(50-200{ }^{\circ} \mathrm{C}\right)$, 


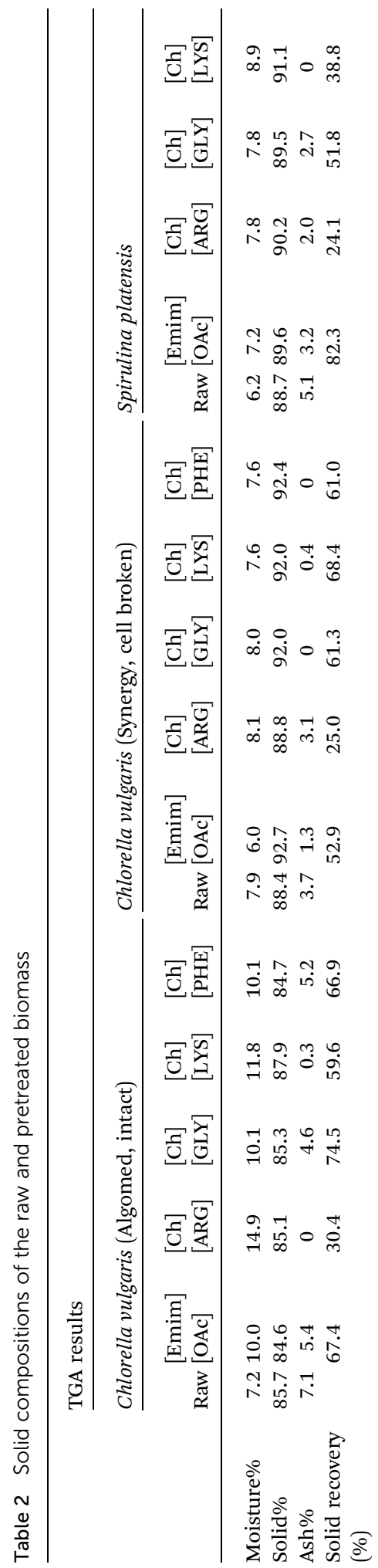




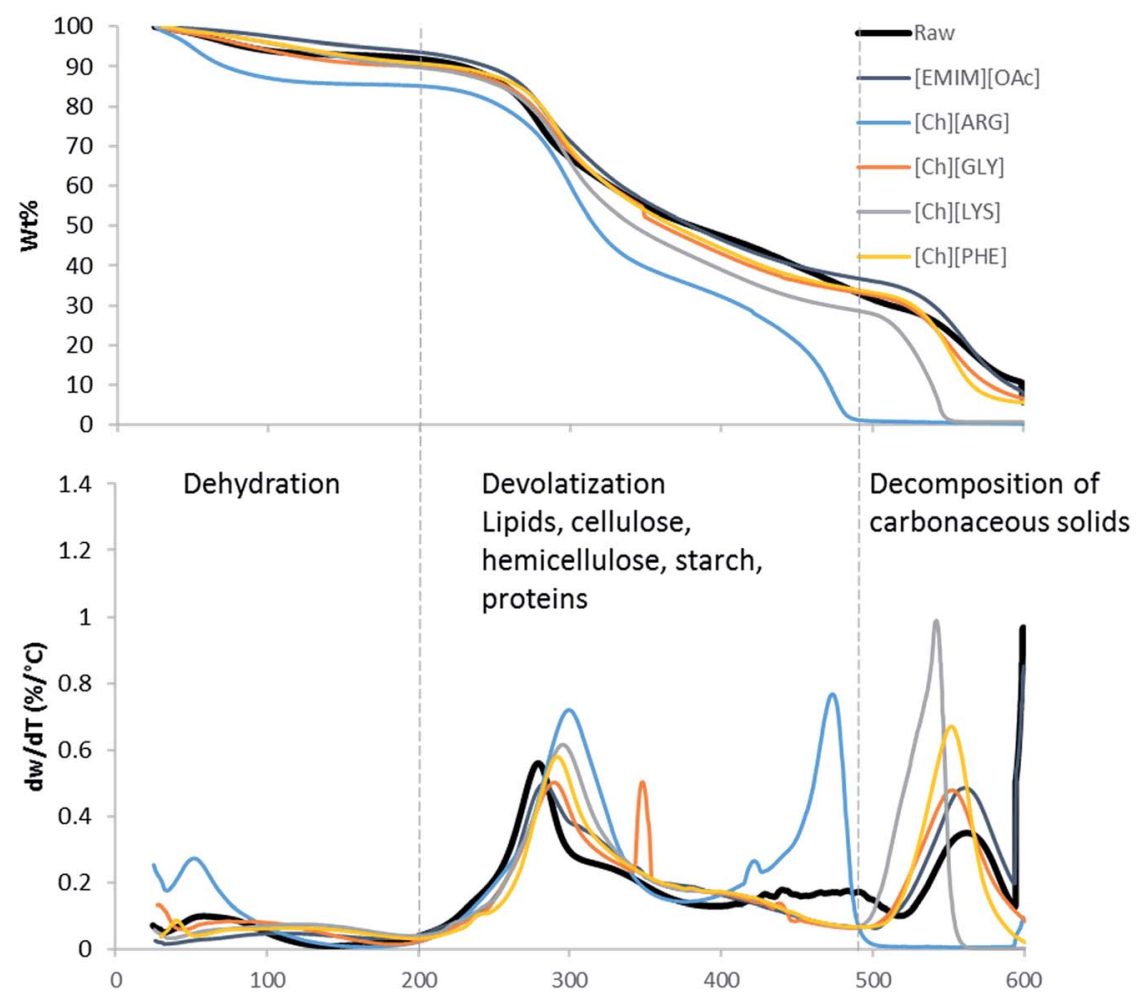

Fig. 1 TGA profiles and their first derivative curves for intact C. vulgaris and its treated counterparts.

devolatization of the hydrocarbon chains of the lipids, cellulose, hemicellulose, starch and proteins $\left(200-500{ }^{\circ} \mathrm{C}\right)$, and decomposition of the carbonaceous solids (500-600 ${ }^{\circ} \mathrm{C}$ ). These finding are similar to the reported TGA profiles of green algae. ${ }^{41}$ Fig. 1-3 show that after pretreatment with ILs the third decomposition stage shifts to a lower temperature, signifying fragmentation of the biomass to lower molecular weight molecules. The largest shift in all three algae samples can be seen for [Ch][ARG] (Fig. 1-3, blue line), signifying that this IL was better than all the other ILs in digesting the algal biomass. This compliments the solid recovery results (Table 2), in which [Ch][ARG] pretreatment resulted in the lowest solid recovery (30.4\% for intact C. vulgaris, $25.0 \%$ for broken C. vulgaris and $24.1 \%$ for $S$. platensis).

There is a consistent reduction of ash in all of the pretreated biomass (see Table 2) samples. Ash is composed of minerals together with silica, which can be dissolved using alkali solution. Hence it is not surprising that some of the ash was lost during the pretreatment, since the IL solutions were all basic.

\section{Effect of the pretreatment on algal lipids}

The two Chlorella vulgaris samples had similar lipid contents (287.9 and $302.8 \mathrm{mg} \mathrm{g}^{-1}$ total lipids/dry solid) while the $S$. platensis strain only had 


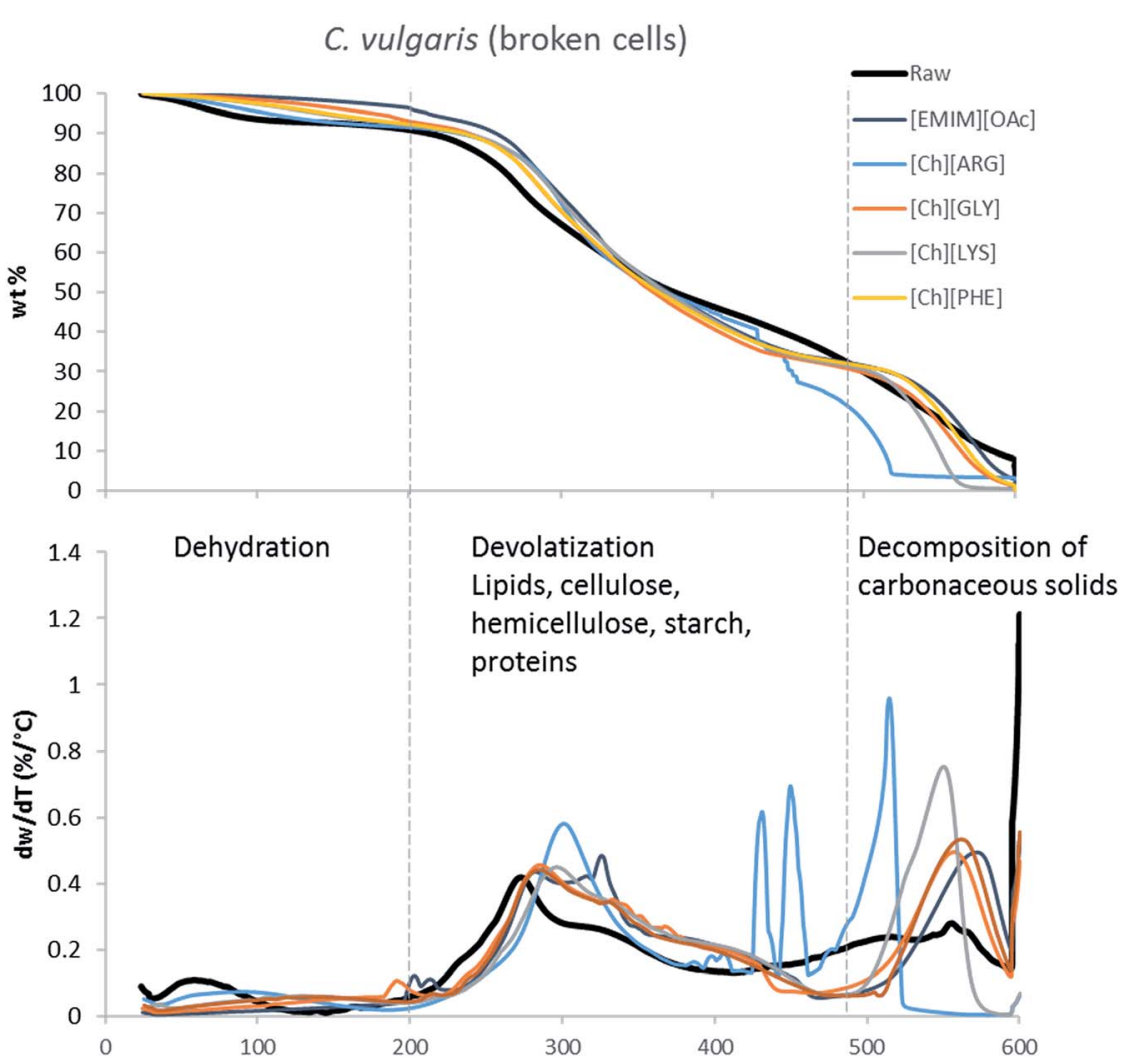

Fig. 2 TGA profiles and their first derivative curves for broken C. vulgaris and its treated counterparts.

102.7 $\mathrm{mg} \mathrm{g}^{-1}$ (see Table 3). C18 long fatty acids constitute the majority of the lipids in all three samples. Pretreatment of the algae with ILs resulted in a partial dissolution of the lipids into the IL solutions. The lipid content of the solid residues ranged widely from $5.8 \%$ to $88.3 \%$ of the starting values. [Ch][ARG] was the most effective IL, leaving behind only $5.8 \%$ (intact $C$. vulgaris), $12.5 \%$ (broken C. vulgaris) and $46.3 \%$ (S. platensis) of the starting lipids. In comparison, [Emim][OAc] was less efficient. It dissolved lipids for the intact C. vulgaris $(13.1 \%$ lipids remaining in the solid), but not as effectively for the broken forms of $C$. vulgaris $(58.3 \%)$ and the $S$. platensis $(88.3 \%)$. The other three choline-amino acid ILs behaved similarly to each other, working well for the broken form of $C$. vulgaris $(18.8-24.5 \%)$, and moderately for the intact C. vulgaris $(32.0-67.0 \%)$ and S. platensis (45.2 and 57.3\%) (note: S. platensis was not tested with [Ch][PHE] because a preliminary experiment showed poor performance). It is worth noting that $[\mathrm{Ch}][\mathrm{ARG}]$ and $[\mathrm{Emim}][\mathrm{OAc}]$ dissolved more lipids from the intact $C$. vulgaris than the broken form, which is unusual since the intact $C$. vulgaris possesses a strong cell wall that should inhibits dissolution. Disparity in the sample sources might account for this abnormality; or the process of cell wall breaking by heating could have destroyed some lipids that if left intact could have been solubilized by 


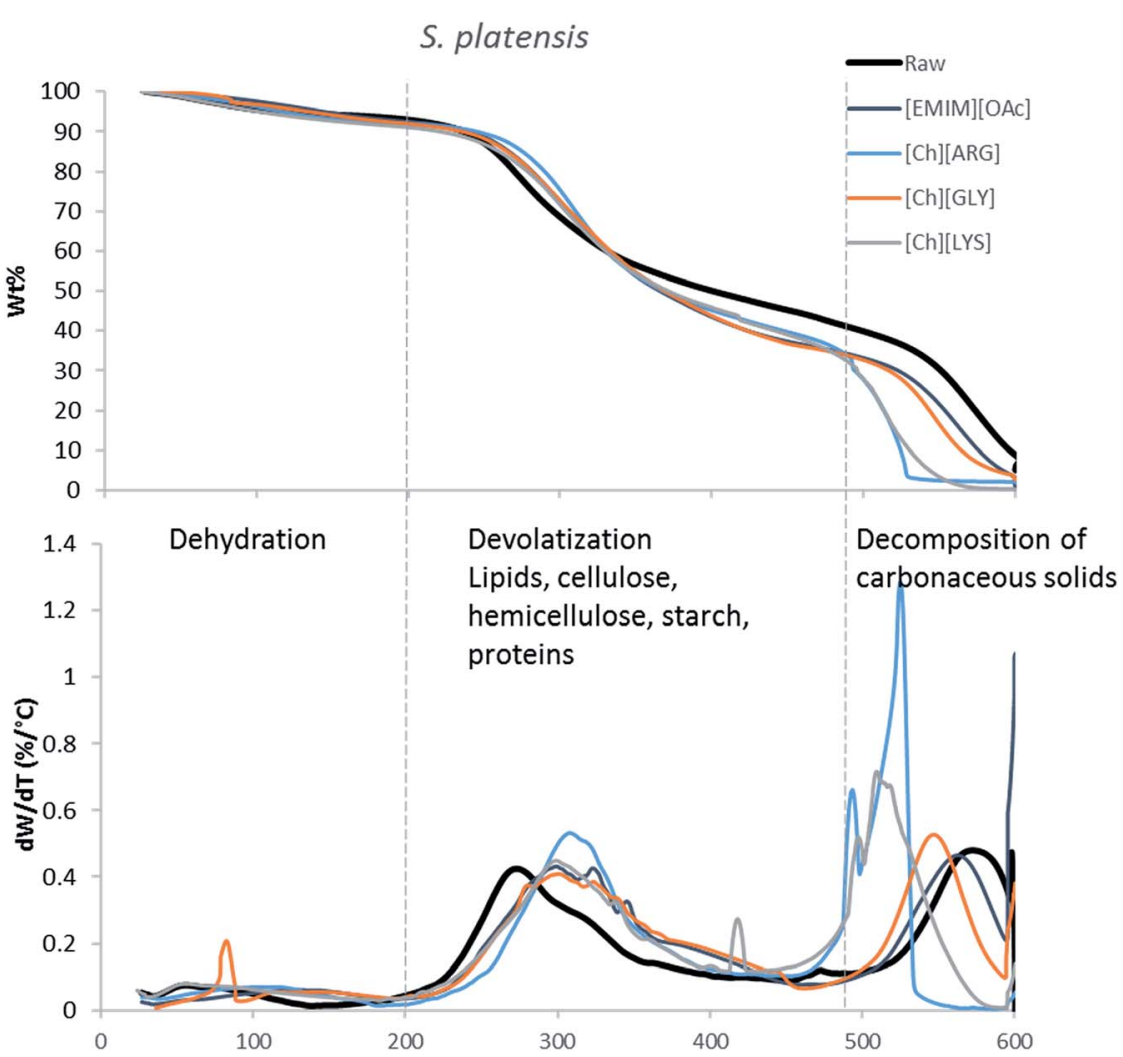

Fig. 3 TGA profiles and their first derivative curves for S. platensis and its treated counterparts.

the ILs studied. Regardless of the sample type, it is clear that [Ch][ARG] was superior over other ILs in removing lipids from the samples of C. vulgaris.

The second step of retrieving the lipids involves extracting them from the IL liquor using hexane. The IL liquors were neutralized with acid, and the lipids were extracted using hexane. Without neutralization, no fatty acids were recovered from the IL liquors. Neutralization allows the fatty acids to convert to their neutral form, which is more soluble in hexane than the anionic form. The purity of the crude lipid extracts was analyzed using GC and was found to vary between 20 and $100 \%$. These values were taken into account to calculate the actual lipid yield. In cells, lipids are stored as triacylglycerols in free form and also in lipid droplets, surrounded by a phospholipid monolayer decorated with proteins. For successful extraction of lipids, pretreatment must break down the cell wall and the cell membrane, as well as release the lipids from any bound proteins. It was found that not all the lipids that were removed from the biomass could be extracted using the hexane. Fig. 4 displays the distribution of lipids after pretreatment. [Ch] [ARG] further displays its superiority over the other ILs in disintegrating lipids, as the hexane was able to extract $10.0 \%$ (intact C. vulgaris), $33.6 \%$ (broken C. vulgaris) and $51.1 \%$ (S. platensis) of the total lipids (Table 1 and Fig. 4, orange bars). It is also evident that the broken form of $C$. vulgaris yielded more lipid than the intact 


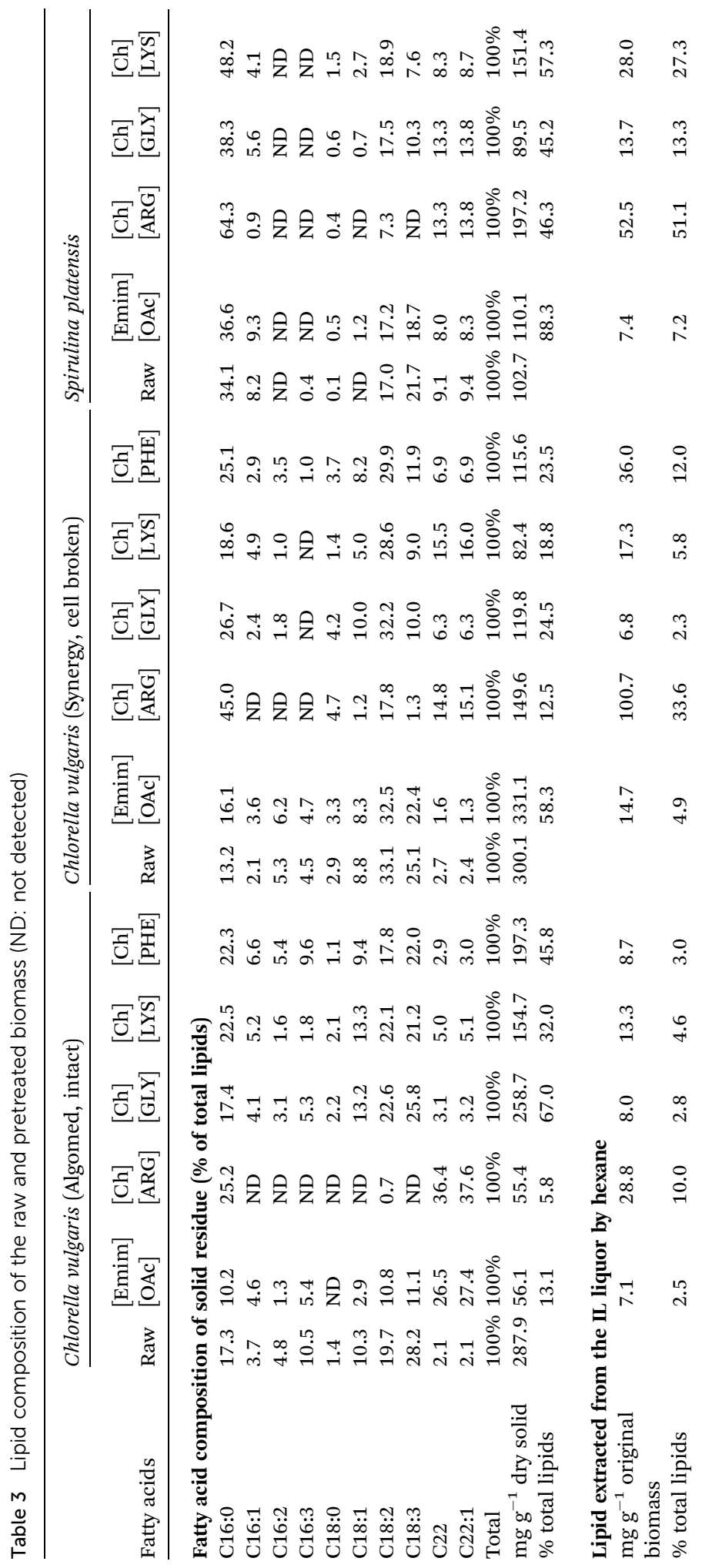




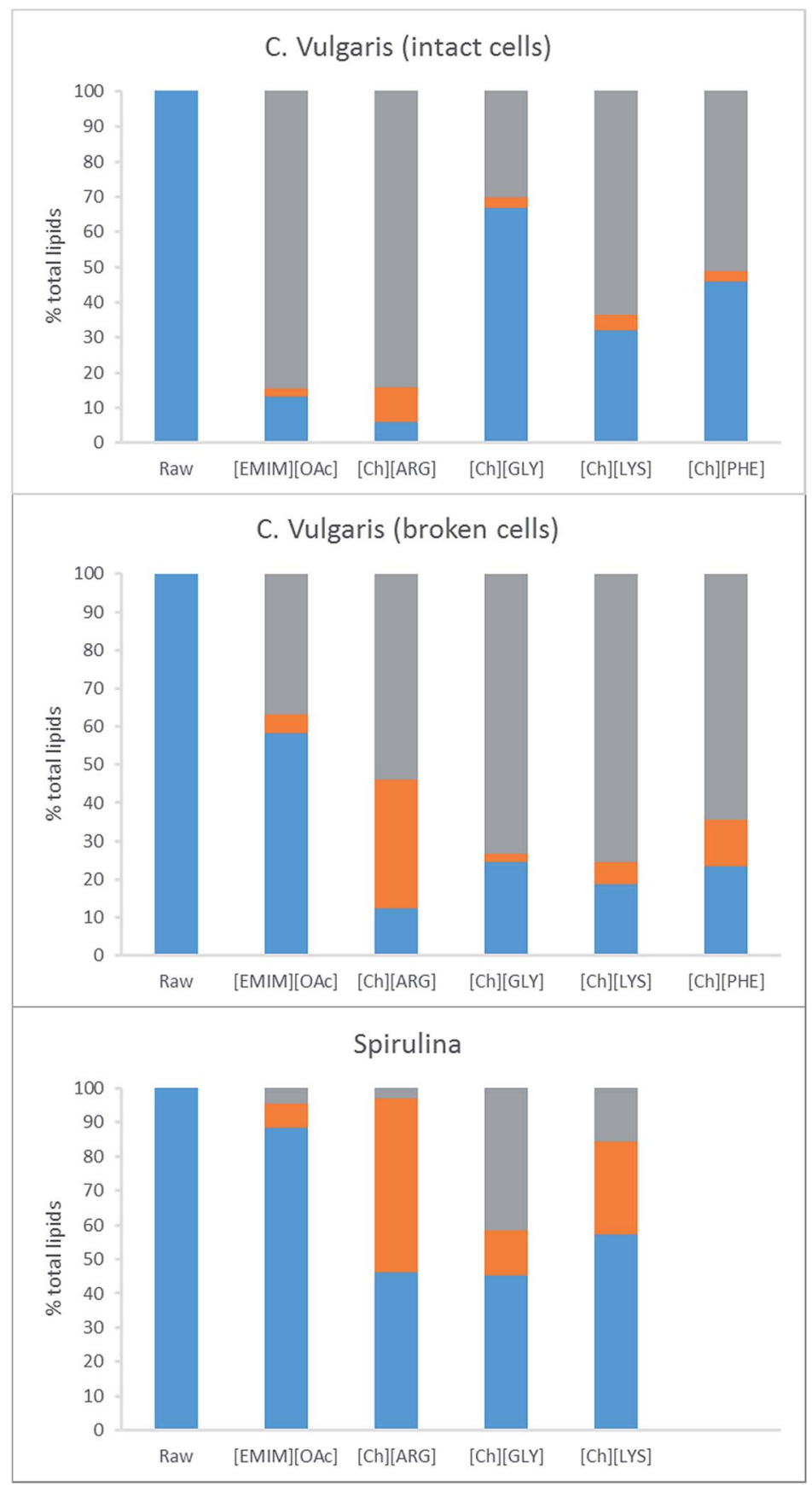

Fig. 4 Distribution of lipids after pretreatment and extraction. Blue bar: \% lipids remaining in solid, orange bar: \% lipids extractable using hexane, grey bar: \% lipids remaining in liquor unextractable using hexane. 
form $(33.6 \%$ compared to $10.0 \%)$, despite initially dissolving less into the IL liquor. This implies that the process of cell wall breaking does help downstream lipid extraction, allowing the IL to penetrate the cells and disintegrate lipids better. The pros and cons of the cell wall breaking pretreatment are discussed in the last section.

For the other ILs, the majority of the dissolved lipids stayed in the liquor, possibly due to the lipids still being bound within the lipid droplets, or bound to proteins, unextractable using hexane. Hexane was able to extract $2.5 \%, 4.5 \%$ and $7.2 \mathrm{wt} \%$ (intact $C$. vulgaris, broken C. vulgaris and S. platensis) of the total lipids out of the $[\mathrm{Emim}][\mathrm{OAc}]$ liquors. These values are lower than the reported values for [Emim] [OAc] pretreatment with $C$. vulgaris using other methods. ${ }^{24,25,28}$ It is worth noting that the extraction time was short (vigorous shaking followed by immediate centrifugation to separate the hexane layer), unlike other methods in which the IL/biomass mixtures were stirred with hexane for hours. ${ }^{25}$ Intact $C$. vulgaris proved difficult for all but [Ch][ARG], extracting yield for [Emim][OAc] and the other three amino acid ILs were all less than $5 \%$. Significant yields were noted for [Ch][LYS]/S. platensis (27.1\%) and [Ch][PHE]/broken C. vulgaris (12.0\% yield).

One possible explanation for the effectiveness of [Ch][ARG] is the structure of the anion, as compared to the other IL anions. Studies have found that in applications involving ILs, the anion plays an important role in dissolving substrates, much more so than the cation. ${ }^{22}$ In lignocellulosic biomass pretreatment, the ILs that worked well all contained good $\mathrm{H}$-bond acceptor anions e.g. $\mathrm{Cl}^{-}$ or $\mathrm{OAc}^{-22,40,42}$ that allow the ILs to bind to $-\mathrm{OH}$ groups that are present in the cellulose and proteins in the biomass. All amino acid anions have at least 3 hydrogen bonding sites, one from the amino $\mathrm{NH}_{2}$ and two from the carboxylate $\mathrm{COO}^{-}$. In this study, the argininate anion has three extra nitrogens on its branch, allowing a total of 6 hydrogen bonding sites to bind to the biomass. [Ch][LYS] also has one extra nitrogen on the anion, which might explain its better performance than [Ch][GLY]. [Ch][PHE] does not have any extra hydrogen bonding sites, but does have an aromatic phenyl ring, which suggests that $\pi-\pi$ stacking might be playing a role in the interaction between $[\mathrm{Ch}][\mathrm{PHE}]$ and the $C$. vulgaris cells.

\section{Effect of the pretreatment on carbohydrates}

Carbohydrate composition of the raw biomass and their pretreated counterparts was determined using a two-step acid hydrolysis according to the NREL protocol. Previous studies on the carbohydrate content of algal biomass showed that the two most abundant monomeric sugars present in C. vulgaris are D-galactose and Dglucose (49 wt $\%$ and $35 \mathrm{wt} \%$ of total carbohydrate respectively). ${ }^{43}$ The ACCU-CHEK Performa glucose meter used was responsive to both D-glucose and D-galactose. This is not unusual, for several glucose-meters have been reported to give unusually high readings in the presence of other sugars such as maltose or galactose. ${ }^{44}$ While this is not desirable in a clinical setting, it conveniently provided us with a quick way to measure multiple sugars simultaneously. Our calibration indicated that this meter is responsive almost equally to glucose and galactose, and measurement of 5 different mixtures of glucose and galactose produced readings that were all within $10 \%$ error of the total sugar content. Hence readings are efficiently the sum of D-glucose and Dgalactose, which conveniently constitutes $84 \mathrm{wt} \%$ of the total carbohydrate present in the algal biomass. 


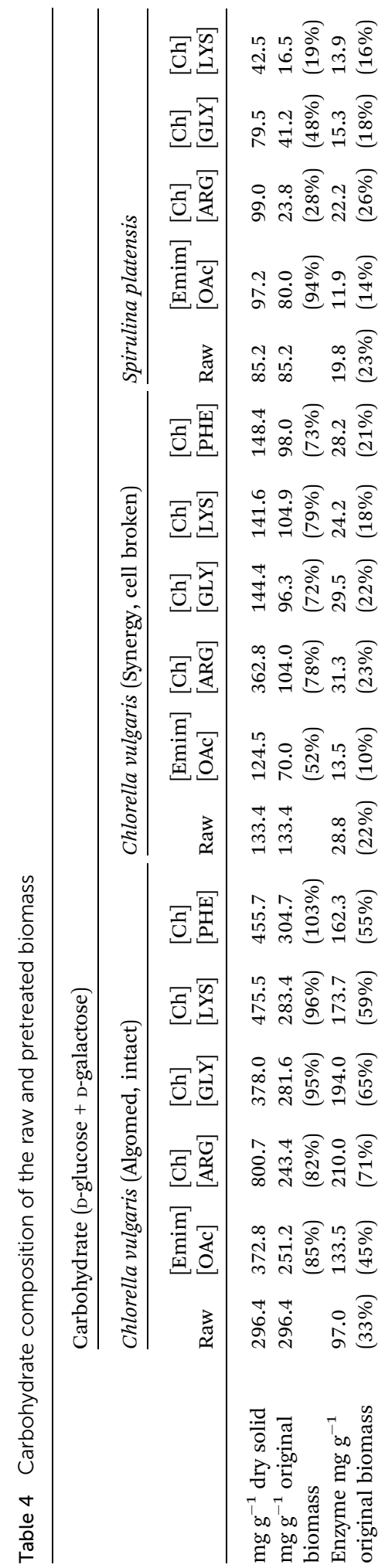


C. vulgaris contained $296.4 \mathrm{mg}$ carbohydrate per $\mathrm{g}$ of biomass (intact form), $133.4 \mathrm{mg} \mathrm{g}^{-1}$ (broken form) while $S$. platensis contained only $85.2 \mathrm{mg} \mathrm{g}^{-1}$ (Table 4 ). $S$. platensis is consumed as a dietary fibre supplement, credited to its fibrous and low calorie nature, in line with its lows lipid (102.7 $\left.\mathrm{mg} \mathrm{g}^{-1}\right)$ and low sugar compositions. Pretreatment of $C$. vulgaris (both samples) produced solids richer in carbohydrates. Most exceptional was [Ch][ARG] which produced $800.7 \mathrm{mg} \mathrm{g}^{-1}$ carbohydrate rich solid (equivalent to $82 \%$ starting sugars when solid recovery of $30.4 \%$ is taken into account). Combined with the lipid value, it is evident that this IL had carried out a highly precise fractionation of the biomass, for it has selectively dissolved the lipids of the biomass to produce a sugar rich solid. The effect was much more pronounced for the intact form of $C$. vulgaris than the broken form $\left(362.8 \mathrm{mg} \mathrm{g}^{-1}\right.$ sugar per dry solid residue), likely due to the carbohydrate storage (mostly as starch) in the broken cells being partially destroyed in the process of cell-wall breaking, consequently allowing free sugars to dissolve into the aqueous solution of the IL liquors. Other ILs produced solids less rich in carbohydrates than [Ch][ARG], but still retained $85-103 \%$ starting sugars from the intact C. vulgaris, and $52-79 \%$ from the broken $C$. vulgaris. It is clear that more sugar was lost from the broken C. vulgaris than the intact form. By contrast, in the case of $S$. platensis, only pretreatment with [Emim][OAc] preserves the sugar in the solid (94\% sugar retained), choline-amino acid pretreatment resulted in a large loss of sugars. S. platensis has a softer cell wall than C. vulgaris, which must have allowed the sugars to dissolve into the aqueous solution of the choline amino acid ILs.

The bioethanol production potential of raw biomass and their pretreated counterparts was estimated using enzymatic hydrolysis. A concoction of $\alpha$ amylase, $\alpha$-amyloglucosidase and cellulase was used to digest the biomass (as carbohydrates are present in both cellulose and starch). The amount ofD-glucose and $\mathrm{D}$-galactose released per $\mathrm{g}$ of the original biomass (i.e. loss of solid during the pretreatment step was taken into account) is reported in Table 4 and Fig. 5. Unlike lignocellulosic biomass, which usually has a very high resistance to enzymes, the raw algal biomass did produce sugar upon enzyme hydrolysis, albeit not

\section{Enzymatic sugar yield}

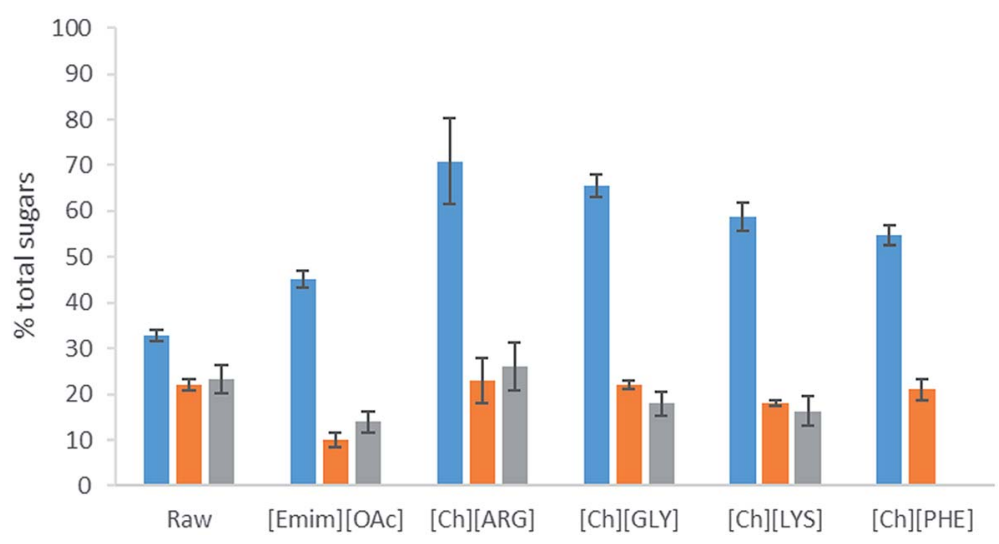

Fig. 5 Enzymatic sugar yield (D-glucose + D-galactose) of the pretreated biomass. Blue bar: C. vulgaris (intact cells), orange bar: C. vulgaris (broken cells), grey bar: S. platensis. $N=$ 2 for all samples. 
completely. Pretreatment was beneficial to the intact form of C. vulgaris, for all the IL pretreatment helped produce more sugar. [Ch][ARG] and [Ch][GLY] were exceptional and helped produce more than double the amount of sugars (released $71 \%$ and $65 \%$ of total sugars) as compared to the untreated sample $(33 \%)$. On the other hand, for the broken form of $C$. vulgaris and S. platensis, there was not much improvement. This could be explained by the loss of sugar to the aqueous solution of the ILs. However, it is not clear why [Emim][OAc] pretreated S. platensis showed such a low activity towards enzyme hydrolysis, despite being richer in carbohydrate. [Emim][OAc] might have carried out inadequate fractionation, in which only the outer parts of the algae were removed while the core cell structure was still intact and resistant to enzymes; or there might have been strong attachment of the [Emim][OAc] molecules to the algal biomass which consequently poisoned and diminished the enzyme activity.

\section{Factors to consider for commercialization - comparing the behaviour of intact and broken $C$. vulgaris}

Model studies by Haas et al. had shown that dewatering and drying of biomass constitutes $70 \%$ of the energy required for biodiesel production from algal biomass. ${ }^{45}$ As the choline-amino acid ILs used in this work are aqueous solutions this means dried algae is not required. Furthermore, simple synthesis and cheap starting materials are certainly advantageous in terms of commercial viability. Recycling of the ILs is likely possible (though not investigated yet in this paper). We envisage addition of an alkali (e.g. $\mathrm{NaOH})$ to neutralize the added acid followed by the removal of excess water will effect recycling of the IL. Accumulation of biomass residuals in the ILs might occur after repeated cycles and its consequence will need to be examined.

The question as to whether physical pretreatment is required remains open. As [Ch][ARG] produced the best results both in terms of lipids and sugar yields, it will be used to discuss the pros and cons of physical pretreatment. Cell disruption techniques for C. vulgaris (e.g. using pressure, ultrasonication, ozonation ${ }^{46}$ or enzymes ${ }^{47}$ ) certainly demand energy. Intact $C$. vulgaris fractionated better than broken ones, for $5.8 \%$ of total lipids and $82 \%$ of total sugars remained in the solid, $c f .12 .5 \%$ and $78 \%$ for broken $C$. vulgaris. Enzymatic hydrolysis results were also better for the pretreated intact $C$. vulgaris (71\% of total sugars was released compared to only $23 \%$ from the broken cells). However, the dissolved lipids of intact C. vulgaris were not easily recovered using hexane (only 10.0\% was extracted, compared to $33.6 \%$ from the broken cells). Nevertheless, optimization of the extracting technique (e.g. longer immersion time with hexane, or addition of methanol as an anti-solvent instead of acid) might help improve the lipid yield. Taking everything into account, our opinion is that using intact cells and skipping any physical treatment offers a more economical solution. Optimization of the extraction step has to be investigated in future work.

\section{Conclusion}

We have demonstrated the use of a series of low cost ILs and [Emim][OAc] for the pretreatment of algal biomass. Among the highlights are the simple synthesis of the choline amino acids, the use of aqueous solutions (65\% IL to $35 \%$ water) 
instead of neat ILs, mild pretreatment conditions $\left(70^{\circ} \mathrm{C}, 3 \mathrm{~h}\right)$, high loading $(20 \mathrm{w} / \mathrm{v} \%)$ and extraction of up to $51.1 \%$ of total lipids. Most exceptional was choline argininate, which yielded the most lipids and sugars. The structure of the argininate anion is likely responsible for its effectiveness, for it contains 6 hydrogen-bonding sites to interact with the biomass. Along with lipids, ash was also removed during the pretreatment, due to the basicity of the IL solutions dissolving the silica in the ash. Our results show physical pretreatment of C. vulgaris, to break open the cell wall prior to chemical pretreatment, is not necessary. While physical pretreatment does yield more lipids, the additional energy demand, reduction in fractionation efficiency and lower sugar yield are unfavourable. Simple optimization of the extraction step (such as longer soaking time with hexane or using $\mathrm{MeOH}$ as an antisolvent) might help maximize the yield of lipid when the intact $C$. vulgaris are used. Recycling of the ILs and optimization of the extraction step would be the subject of further study.

\section{Acknowledgements}

The authors acknowledge the financial support provided by the Priority Research Centre for Advanced Fluids and Interfaces, the University of Newcastle, Australia. Rob Atkin thanks the Australian Research Council for a Future Fellowship. The work by the DOE Joint BioEnergy Institute (http://www.jbei.org/) is supported by the DOE Office of Science, Office of Biological and Environmental Research, through contract DE-AC02-05CH11231 between Lawrence Berkeley National Laboratory and the DOE.

\section{References}

1 A. Singh, P. S. Nigam and J. D. Murphy, Bioresour. Technol., 2011, 102, 10-16.

2 R. Halim, M. K. Danquah and P. A. Webley, Biotechnol. Adv., 2012, 30, 709-732.

3 M. K. Lam and K. T. Lee, Biotechnol. Adv., 2012, 30, 673-690.

4 R. R. Kumar, P. H. Rao and M. Arumugam, Front. Energy Res., 2015, 2, 1-9.

5 J. J. Milledge, Rev. Environ. Sci. Biotechnol., 2011, 10, 31-41.

6 C. X. Liu, S. Subashchandrabose, H. Ming, B. Xiao, R. Naidu and M. Megharaj, J. Appl. Phycol., 2016, 28, 3331-3341.

7 C. X. Liu, S. R. Subashchandrabose, M. Megharaj, Z. Q. Hu and B. Xiao, Bioresour. Technol., 2016, 218, 1170-1177.

8 C. H. Hsieh and W. T. Wu, Bioresour. Technol., 2009, 100, 3921-3926.

9 G. Van Vooren, F. Le Grand, J. Legrand, S. Cuine, G. Peltier and J. Pruvost, Bioresour. Technol., 2012, 124, 421-432.

10 A. L. Ahmad, N. H. M. Yasin, C. J. C. Derek and J. K. Lim, Renewable Sustainable Energy Rev., 2011, 15, 584-593.

11 D. S. Domozych, M. Ciancia, J. U. Fangel, M. D. Mikkelsen, P. Ulvskov and W. G. T. Willats, Front. Plant Sci., 2012, 3, 82.

12 A. M. Aboshady, Y. A. Mohamed and T. Lasheen, Biol. Plant., 1993, 35, 629-632.

13 E. Kapaun, E. Loos and W. Reisser, Phytochemistry, 1992, 31, 3103-3104.

14 E. G. Bligh and W. J. Dyer, Can. J. Biochem. Physiol., 1959, 37, 911-917.

15 A. R. Byreddy, A. Gupta, C. J. Barrow and M. Puri, Mar. Drugs, 2015, 13, 51115127. 
16 J. R. McMillan, I. A. Watson, M. Ali and W. Jaafar, Appl. Energy, 2013, 103, 128134.

17 H. C. Greenwell, L. M. Laurens, R. J. Shields, R. W. Lovitt and K. J. Flynn, J. R. Soc., Interface, 2010, 7, 703-726.

18 L. M. L. Laurens, N. Nagle, R. Davis, N. Sweeney, S. Van Wychen, A. Lowell and P. T. Pienkos, Green Chem., 2015, 17, 1145-1158.

19 J. P. Hallett and T. Welton, Chem. Rev., 2011, 111, 3508-3576.

20 N. V. Plechkova and K. R. Seddon, Chem. Soc. Rev., 2008, 37, 123.

21 H. Olivier-Bourbigou, L. Magna and D. Morvan, Appl. Catal., A, 2010, 373, 1-56.

22 A. Brandt, J. Grasvik, J. P. Hallett and T. Welton, Green Chem., 2013, 15, 550583.

23 A. A. Elgharbawy, M. Z. Alam, M. Moniruzzaman and M. Goto, Biochem. Eng. J., 2016, 109, 252-267.

24 V. C. A. Orr and L. Rehmann, Current Opinion in Green and Sustainable Chemistry, 2016, 2, 22-27.

25 S.-A. Choi, J.-S. Lee, Y.-K. Oh, M.-J. Jeong, S. W. Kim and J.-Y. Park, Algal Res., 2014, 3, 44-48.

26 Y. H. Kim, S. Park, M. H. Kim, Y. K. Choi, Y. H. Yang, H. J. Kim, H. Kim, H. S. Kim, K. G. Song and S. H. Lee, Biomass Bioenergy, 2013, 56, 99-103.

27 Y. H. Kim, Y. K. Choi, J. Park, S. Lee, Y. H. Yang, H. J. Kim, T. J. Park, Y. H. Kim and S. H. Lee, Bioresour. Technol., 2012, 109, 312-315.

28 S. A. Choi, Y. K. Oh, M. J. Jeong, S. W. Kim, J. S. Lee and J. Y. Park, Renewable Energy, 2014, 65, 169-174.

29 M. Olkiewicz, M. P. Caporgno, J. Font, J. Legrand, O. Lepine, N. V. Plechkova, J. Pruvost, K. R. Seddon and C. Bengoa, Green Chem., 2015, 17, 2813-2824.

30 V. C. A. Orr, N. V. Plechkova, K. R. Seddon and L. Rehmann, ACS Sustainable Chem. Eng., 2016, 4, 591-600.

31 J. M. Restrepo-Florez, A. Bassi, L. Rehmann and M. R. Thompson, Bioresour. Technol., 2013, 147, 456-463.

32 K. Gao, V. Orr and L. Rehmann, Bioresour. Technol., 2016, 206, 77-85.

33 L. B. Malihan, G. M. Nisola, N. Mittal, J. G. Seo and W. J. Chung, Renewable Energy, 2014, 66, 596-604.

34 T. J. Trivedi and A. Kumar, Green Sustainable Chem., 2014, 4, 190-201.

35 M. J. Liszka, A. Kang, N. V. S. N. M. Konda, K. Tran, J. M. Gladden, S. Singh, J. D. Keasling, C. D. Scown, T. S. Lee, B. A. Simmons and K. L. Sale, Green Chem., 2016, 18, 4012-4021.

36 S. V. Wychen, K. Ramirez and L. M. L. Laurens, Determination of Total Lipids as Fatty Acid Methyl Esters (FAME) by in situ Transesterification, National Renewable Energy Laboratory, 2013.

37 S. V. Wychen and L. M. L. Laurens, Determination of total carbohydrates in algal biomass, National Renewable Energy Laboratory, 2015.

38 M. Selig, N. Weiss and Y. Ji, Enzymatic Saccharification of Lignocellulosic Biomass, Report NREL/TP-51042629, National Renewable Energy Laboratory, 2008.

39 STA-20 Starch Assay Kit (Amylase/Amyloglucosidase Method), Sigma.

40 A. Brandt, M. J. Ray, T. Q. To, D. J. Leak, R. J. Murphy and T. Welton, Green Chem., 2011, 13, 2489-2499.

41 K. Kebelmann, A. Hornung, U. Karsten and G. Griffiths, Biomass Bioenergy, 2013, 49, 38-48. 
42 K. Dong, S. J. Zhang and J. J. Wang, Chem. Commun., 2016, 52, 6744-6764.

43 D. W. Templeton, M. Quinn, S. Van Wychen, D. Hyman and L. M. L. Laurens, J. Chromatogr. A, 2012, 1270, 225-234.

44 T. G. Schleis, Pharmacotherapy, 2007, 27, 1313-1321.

45 M. J. Haas, A. J. McAloon, W. C. Yee and T. A. Foglia, Bioresour. Technol., 2006, 97, 671-678.

46 Y. Huang, A. Hong, D. Zhang and L. Li, Environ. Technol., 2014, 35, 931-937.

47 H. G. Gerken, B. Donohoe and E. P. Knoshaug, Planta, 2013, 237, 239-253. 